\title{
A tailored, multifaceted programme in capitation-based family practices improved appropriate use of preventive care manoeuvres
}

\author{
Lemelin J, Hogg W,Baskerville N. Evidence to action: a tailored multifaceted approach to changing family physician \\ practice patterns and improving preventive care. CMAJ 2001 Mar 20;164:757-63.
}

QUESTION: Can a multifaceted intervention programme using nurse facilitators improve preventive care (increased rates of recommended preventive care manoeuvres and decreased rates of non-recommended manoeuvres) in capitation-based family medicine practices?

\section{Design}

Randomised \{allocation concealed*\} $\uparrow$, unblinded,* controlled trial with 18 months of follow up.

\section{Setting}

46 capitation-based health services organisations (HSOs) in family practice settings in Ontario, Canada.

\section{Practices}

46 HSOs were allocated, and 45 were analysed $(64 \%$ group practices, $53 \%$ university affiliated, mean of 3 physicians per practice, and mean age of patients $47 \mathrm{y}$ ).

\section{Intervention}

23 HSOs were allocated to each intervention. Intervention HSOs were assigned to 1 of 3 nurse facilitators. The nurse made several visits and worked with the practice physicians and staff as a facilitator to adopt a 7-component programme to each practice's needs and wishes. The intervention strategies were designed to improve preventive care performance by using audit and ongoing feedback, consensus building, opinion leaders and networking, academic detailing with educational materials, reminder systems, patient mediated activities, and patient education materials. The evaluation was based on 8 recommended and 5 inappropriate preventive manoeuvres. 1 intervention HSO was lost to follow up.

\section{Main outcome measure}

Preventive performance was calculated as the proportion of eligible patients who received recommended manoeuvres (up-to-dateness) minus the proportion of patients who received inappropriate preventive manoeuvres (inappropriateness).

\section{Main results}

At baseline, the groups had similar scores. Preventive performance increased from $32 \%$ at baseline to $43 \%$ at 18 months in the intervention HSOs and remained unchanged at $32 \%$ in the control HSOs ( $\mathrm{p}$ for difference $<0.001)$. Up-to-dateness scores for the intervention HSOs increased from $52 \%$ to $62 \%$ during the intervention. Corresponding scores for the control HSOs were $55 \%$ and $57 \%$ (p for difference $<0.01$ ). Inappropriateness scores decreased slightly in the intervention HSOs (from 21\% to 19\%) and increased in the control HSOs $(23 \%$ to $26 \%$ ) ( $\mathrm{p}$ for difference $<0.05$ ). Preventive performance scores for the intervention HSOs showed successive increases at 9, 15, and 18 months. For individual manoeuvres, intervention HSOs completed more influenza vaccinations, counselled more young women about folic acid, and had a decrease in inappropriateproteinuria and blood glucose screening testing ( $\mathrm{p}$ for difference $<0.05$ ).

\section{Conclusion}

A nurse facilitator who helped individualise and implement a multifaceted programme to improve the appropriate use of preventive care manoeuvres in health service organisations in family practice settings was successful.

*See glossary.

$\dagger$ Information provided by author.

\section{COMMENTARY}

Changing physician practice is not easy. Passive interventions (eg, lectures, journals, or mailed guidelines) rarely work. Interventions that use several strategies tailored to individual practice needs, as in the study by Lemelin $e t a l$, are more likely to be effective. ${ }^{1}$ In addition, use of an external facilitator to work with practice personnel in making these changes has been effective in other studies in various primary care settings.

Only modest changes in prevention rates were noted in the study. However, the progressive increase in preventive performance over time and the differential effects on recommended and inappropriate manoeuvres suggest that the improvements signalled real and progressive changes in practice patterns rather than a general increase in awareness of prevention because of the visits of the study nurse. In other studies, such practice changes have persisted for up to 2 years after the facilitator intervention ended. ${ }^{2}$

Achieving these modest changes required a large effort. Study nurses made an average of 33 visits of 1 to 2 hours to each practice. However, volunteers from such organisations as the American Cancer Society can be trained in this role." Alternatively, because the actual practice changes are not complicated, a motivated physician could (in theory) make them without an external support person by using such materials as those from the Put Prevention into Practice programme of the US Public Health Service.

Lorne A Becker, MD

State University of New York Upstate Medical University Syracuse, New York, USA

1 Bero LA, Grilli R, Grimshaw JM, et al. Closing the gap between research and practice: an overview of systematic reviews of interventions to promote the implementation of research findings. The Cochrane Effective Practice and Organization of Care Review Group. BMJ 1998;317:465-8

2 Dietrich AJ, Sox CH, Tosteson TD, Woodruff CB. Durability of improved physician early detection of cancer after conclusion of intervention support. Cancer Epidemiol Biomarkers Prev 1994;3:335-40.

3 Woodruff CB, Dietrich AJ, Carney PA, et al. Volunteer facilitators assist community practices with enhancing cancer control. Arch Fam Med 1996;5:560-5.

4 http://www.ahcpr.gov/clinic/ppipix.htm. Accessed June 1, 2001 